\title{
Conservação pós-colheita de pinhões [sementes de Araucaria angustifolia (Bertoloni) Otto Kuntze] armazenados em diferentes temperaturas
}

\author{
Postharvest preservation of 'pinhões' [seeds of Araucaria angustifolia (Bertoloni) Otto Kuntze] stored \\ at different temperatures
}

\author{
Cassandro Vidal Talamini do Amarante ${ }^{\mathrm{I}}$ Clenilso Sehnen Mota ${ }^{\mathrm{II}}$ \\ Clarice Aparecida Megguer ${ }^{\text {III }}$ Gilberto Massachi Ide ${ }^{\mathrm{III}}$
}

\section{RESUMO}

O pinhão (semente de Araucaria angustifolia) representa importante fonte de renda aos produtores rurais $e$ uma opção a mais de alimento característico a ser oferecido aos turistas e à população em geral na região Sul do Brasil. A temperatura e a umidade de armazenamento são os principais determinantes da preservação pós-colheita de pinhões. Este trabalho objetivou avaliar os efeitos da temperatura de armazenamento nas taxas respiratórias e de evolução de etileno, bem como na perda de massa fresca e na germinação póscolheita de pinhões destinados para consumo alimentar humano. Seguiu-se o delineamento experimental inteiramente casualizado com seis temperaturas de armazenamento (2, 10, $20,30,40$ e $50^{\circ} \mathrm{C}$ ) e quatro repetições, cada repetição correspondendo a amostras contendo cerca de $300 \mathrm{~g}$ de pinhões. Pinhões armazenados nas diferentes temperaturas não apresentaram produção de etileno (em níveis detectáveis através de cromatografia gasosa, com sensibilidade de 1ppm). A taxa respiratória aumentou substancialmente com o aumento na temperatura, com um $Q_{10} \cong 2,5$ na faixa de temperatura de 2 a $37,1^{\circ} \mathrm{C}$. Houve redução substancial da respiração com o aumento na temperatura de 37,1 para $50^{\circ} \mathrm{C}$. A maior germinação dos pinhões foi verificada na temperatura de $20^{\circ} \mathrm{C}$ ( $\sim 55 \%$ dos pinhões germinados aos 26 dias de armazenamento), reduzindo em temperaturas menores ( $1 \%$ e $21 \%$ de germinação nas temperaturas de 2 e $10^{\circ} \mathrm{C}$, respectivamente) ou maiores ( $9 \%$ e $<1 \%$ de germinação nas temperaturas de $30^{\circ} \mathrm{C}$ e $40-50^{\circ} \mathrm{C}$, respectivamente). Estes resultados demonstram a importância do resfriamento de pinhões para temperaturas próximas a $0^{\circ} \mathrm{C}$, visando a reduzir a atividade metabólica, especialmente a respiração, com vistas à preservação de sua qualidade e à redução na perda de massa fresca e na germinação pós-colheita.

Palavras-chave: pinheiro do Paraná, fisiologia da semente, respiração, perda de massa fresca, germinação.

\begin{abstract}
The seeds ('pinhões') of Brazilian pine (Araucaria angustifolia) represent an important sorce of income to the rural comunities and also an additional food product to be offered to the tourists and population in Southern Brazil. Temperature and humidity in the storage environment are the mais factors affecting 'pinhões' postharvest preservation. This work was carried out to evaluate the effects of storage temperature on postharvest respiration and ethylene production, as well as on fresh mass loss and germination of 'pinhões' intended for human consumption. The experiment followed a completely randomized design with six storage temperatures $\left(2,10,20,30,40\right.$, and $\left.50^{\circ} \mathrm{C}\right)$ and four replicates, each replicate with about $300 \mathrm{~g}$ of 'pinhões'. 'Pinhões' stored at the different temperatures did not exhibit ethylene production (at levels detectable by gas chromatography, with a sensibility of 1ppm). The respiratory rates increased substancialy in the range from $2^{\circ} \mathrm{C}$ to $37.1^{\circ} \mathrm{C}$, with a $Q_{10} \cong 2.5$. The respiration decreased substantialy by increasing the temperature from $37.1^{\circ} \mathrm{C}$ to $50^{\circ} \mathrm{C}$. The higher germination rate was achieved for 'pinhões' stored at $20^{\circ} \mathrm{C}$ ( $\sim 55 \%$ germination after 26 days storage). The germination reduced for 'pinhões' stored at temperatures lower ( $\sim 1 \%$ and $21 \%$ of germination for 'pinhões' left at 2 and $10^{\circ} \mathrm{C}$, respectively) or higher ( $9 \%$ and $<1 \%$ of germination for 'pinhões' left at $30^{\circ} \mathrm{C}$ and $40-50^{\circ} \mathrm{C}$, respectively) than $20^{\circ} \mathrm{C}$. These results show the importance of 'pinhões' storage at temperatures close to $0^{\circ} \mathrm{C}$ to reduce the metabolic ativity, especially the respiration, and, therefore, to preserve the product quality and to reduce fresh mass loss and seed germination.
\end{abstract}

Key words: Brazilian pine, seed physiology, respiration, fresh mass loss, germination.

ICentro de Ciências Agroveterinárias (CAV), Universidade do Estado de Santa Catarina (UDESC). Av. Luiz de Camões, 2090, CP 281, 88520-000, Lages, SC, Brasil. E-mail: amarante@cav.udesc.br. Autor para correspondência.

IIPrograma de Pós-graduação em Produção Vegetal, CAV, UDESC, Lages, SC, Brasil. E-mail: a6csm@cav.udesc.br.

IIICurso de Agronomia, CAV, UDESC, Lages, SC, Brasil. E-mail: a2gmi@cav.udesc.br. 


\section{INTRODUÇÃO}

O pinheiro do Paraná [Araucaria angustifolia (Bertoloni) Otto Kuntze] é uma espécie secundária, longeva, que ocorre naturalmente no Brasil, distribuindo-se pelos Estados do Paraná, de Santa Catarina e do Rio Grande do Sul. Aparece também em manchas esparsas na região sul dos Estados de São Paulo, Minas Gerais e nas áreas de altitude elevada do Rio de Janeiro (CARVALHO, 1994). Na região Sul do Brasil, devido ao intenso desmatamento nas últimas décadas, o seu cultivo tem recebido forte incentivo por parte de órgãos governamentais, estaduais e municipais, ligados ao meio ambiente e à agricultura.

O pinheiro do Paraná produz anualmente cerca de 40 pinhas, chegando a atingir até 200 pinhas por planta (CARVALHO, 1994). Quando plantado, árvores isoladas iniciam a produção de pinhões entre 10 e 15 anos; porém, em povoamentos, a produção dáse a partir de 20 anos (CARVALHO, 1994). A maturação da pinha caracteriza-se pelo término do seu desenvolvimento, e ocorre em diferentes épocas, dependendo da variedade e do local. No Brasil, os pinhões são colhidos de março a setembro (CARVALHO, 1994).

O pinhão apresenta grande importância na utilização como alimento e como semente para a produção de mudas. A conservação pós-colheita do pinhão como semente é dificultada pela sua natureza recalcitrante (CORBINEAU et al., 1997; RAMOS et al., 1988; SALMEN-ESPINDOLA et al., 1994; TOMPSETT, 1993), já que ocorre rápida perda de sua viabilidade fisiológica com a redução do grau de umidade (AQUILA \& FERREIRA, 1984; FARRANT et al., 1989; FOWLER et al., 1998). Todavia, esta não é uma limitação para consumo na forma de alimento. Na verdade, a brotação do pinhão representa uma desvantagem, já que sementes brotadas não apresentam valor comercial para consumo humano.

Devido á falta de métodos para a conservação in natura e para o processamento industrial, o pinhão tem sido pouco empregado na culinária brasileira. Apesar de sua importância históricocultural na alimentação das populações na região Sul do Brasil, pouca atenção tem sido dada à pesquisa de métodos que preservem a sua qualidade pós-colheita. Técnicas de conservação e industrialização do pinhão devem ser desenvolvidas para promover a comercialização e o uso do pinhão em outras épocas do ano, além da estação de produção, visando a tornar o seu mercado mais atraente, incentivando a sua extração e comercialização por parte dos produtores rurais (SANTOS et al., 2002). Isso representaria uma fonte alternativa de renda para as comunidades rurais, promovendo assim o cultivo do pinheiro do Paraná e desestimulando o corte ilegal dessa espécie florestal, considerada rara ou ameaçada de extinção (KLEIN, 1993).

Este trabalho objetivou avaliar os efeitos da temperatura de armazenamento nas taxas respiratórias e de evolução de etileno, bem como na perda de massa fresca e na germinação pós-colheita de pinhões destinados para consumo alimentar humano.

\section{MATERIAL E MÉTODOS}

Pinhas de pinheiro do Paraná [Araucaria angustifolia (Bertoloni) Otto Kuntze] foram coletadas de plantas com cerca de 100-140 anos de idade, em uma propriedade rural do município de Lages, SC, em junho de 2003, e transportadas imediatamente para o laboratório de Fisiologia Vegetal, no Centro de Ciências Agroveterinárias (CAV), em Lages, SC. As pinhas foram debulhadas, sendo selecionados pinhões sadios (isentos de ataque de pragas, infecções por patógenos e de danos mecânicos) e de tamanho uniforme. Esses pinhões foram armazenados em câmaras BOD, nas temperaturas de $2,10,20,30,40$ e $50^{\circ} \mathrm{C}$, e umidade relativa de $90 \pm 2 \%$. O experimento seguiu o delineamento inteiramente casualizado, com seis tratamentos (temperaturas) e quatro repetições, cada repetição correspondendo a amostras contendo cerca de $300 g$ de pinhões.

Os pinhões foram mantidos nas diferentes temperaturas de armazenamento durante o período máximo de 96 dias, sendo feitas avaliações periódicas de taxas respiratórias e de evolução de etileno, germinação e perda de massa fresca.

As quantificações das taxas respiratórias e de evolução de etileno foram feitas com um cromatógrafo a gás Varian ${ }^{\circledR}$ (modelo CG 3800), equipado com metanador, detector de ionização de chama e coluna Porapaq N (80 a 100 mesh). As temperaturas do forno, do detector, do metanador e do injetor foram de $45,120,300$ e $110^{\circ} \mathrm{C}$, respectivamente. Os fluxos de $\mathrm{N}_{2}$, $\mathrm{H}_{2}$ e ar utilizados foram de 70,30 e $300 \mathrm{~mL} \mathrm{~min}^{-1}$, respectivamente.

As taxas respiratórias e de evolução de etileno foram calculadas e expressas segundo o sistema internacional de unidades, descrito por BANKS et al. (1995), através da seguinte equação:

$$
r_{j}=\frac{\left(V_{\text {frasco }}-\frac{M}{\rho_{\text {pinhão }}^{20}}\right) \Delta p_{j}^{\Delta t}}{R(T+273,15) M \Delta t}
$$


Onde:

$V_{\text {frasco }}=$ volume do frasco utilizado para a medição da taxa de produção do gás $j$;

$M=$ massa dos pinhões (kg);

$\rho_{\text {pinhão }}^{20}=$ densidade $\left(\mathrm{kg} \mathrm{m}^{-3}\right)$ do pinhão a $20^{\circ} \mathrm{C}$;

$\Delta p_{j}^{\Delta t}=$ diferença na pressão parcial do gás $j$, no intervalo de tempo Ät;

$R=$ constante dos gases perfeitos $\left(8,3143 \mathrm{~m}^{3} \mathrm{~Pa} \mathrm{~mol}^{-1}\right.$

$\left.\mathrm{K}^{-1}\right)$;

$T=$ temperatura $\left({ }^{\circ} \mathrm{C}\right)$;

$\Delta \mathrm{t}=$ diferença de tempo entre as amostragem iniciais e finais do gás $j$.

As taxas respiratórias, quantificadas 24 horas após a colocação dos pinhões nas diferentes temperaturas, foram utilizadas para o ajuste de modelo matemático, relacionando temperatura e respiração, visando calcular o valor do $\mathrm{Q}_{10}$.

A avaliação de germinação (\%) foi feita visualmente, considerando semente brotada aquela que apresentava protrusão visível de radícula através da casca da semente. A perda de massa fresca (\%) foi determinada pela diferença entre a massa inicial e aquela no momento da realização das análises, com o auxílio de uma balança com sensibilidade de 0,001g.

Os dados coletados foram analisados estatisticamente com o programa SAS (1990). As análises de regressão não-linear foram realizadas pelo emprego do procedimento PROC NONLIN. As médias de tratamentos foram comparadas pelo teste LSD ao nível de 5\% de probabilidade.

\section{RESULTADOS E DISCUSSÕES}

Considerando a taxa respiratória 24h após o armazenamento dos pinhões nas diferentes temperaturas, foi possível representar graficamente a relação entre temperatura e atividade respiratória (Figura 1). Segundo o modelo matemático ajustado, a taxa respiratória aumentou até a temperatura de $37,1^{\circ} \mathrm{C}$, diminuindo a seguir. Na faixa de temperatura de 2 a $37,1^{\circ} \mathrm{C}$, obteve-se um $\mathrm{Q}_{10} \cong 2,5$. Houve redução da taxa respiratória com o aumento na temperatura de 37,1 a $50^{\circ} \mathrm{C}$, possivelmente como resultado da desnaturação/ inativação de enzimas e desorganização do sistema de membranas celulares (TAIZ \& ZEIGER, 1998).

Pinhões armazenados nas diferentes temperaturas não apresentaram produção de etileno em níveis detectáveis através de cromatografia gasosa, em equipamento com sensibilidade de 1ppm. Isso demonstra que as sementes de pinheiro do Paraná não só apresentam baixa capacidade de produção de etileno, mas também que a biossíntese deste hormônio não é influenciado pela temperatura de armazenamento. $\mathrm{O}$ etileno é o principal hormônio promotor da maturação e senescência em tecidos de plantas (WILLS et al., 1998). A baixa capacidade de produção de etileno demonstra que este hormônio apresenta reduzido ou nenhum controle sobre a senescência e a perda de viabilidade fisiológica em pinhões.

As menores taxas respiratórias foram observadas em pinhões armazenados nas temperaturas de 2 e $10^{\circ} \mathrm{C}$ (Figura 2). As taxas respiratórias em pinhões armazenados na temperatura de $10^{\circ} \mathrm{C}$ aumentaram sensivelmente a partir de 50 dias de armazenamento, sendo significativamente superiores às taxas respiratórias de pinhões armazenados na temperatura de $2^{\circ} \mathrm{C}$ (Figura 2). As taxas respiratórias foram significativamente maiores na temperatura de $20^{\circ} \mathrm{C}$, em relação às duas temperaturas inferiores, e mantiveramse entre valores de 200 e $300 \mathrm{nmol}$ de $\mathrm{CO}_{2} \mathrm{~kg}^{-1} \mathrm{~s}^{-1}$ (Figura 2). As taxas respiratórias foram bastante elevadas no início do experimento nas temperaturas de 30 e $40^{\circ} \mathrm{C}$ (Figura 2). Na temperatura de $30^{\circ} \mathrm{C}$, houve uma queda na taxa respiratória até os 10 dias de armazenamento, mantendo-se constante até os 20 dias, ocorrendo um aumento gradativo posteriormente. Em pinhões mantidos na temperatura de $40^{\circ} \mathrm{C}$, houve uma redução contínua na taxa de respiração até cerca de 16 dias após o início do armazenamento, sendo esses pinhões eliminados aos 26 dias, devido ao aspecto visual não atrativo (casca com pouco brilho e coloração marrom). A taxa respiratória na temperatura de $50^{\circ} \mathrm{C}$ foi substancialmente menor que na temperatura de $20^{\circ} \mathrm{C}$ já a partir da primeira avaliação, estando os pinhões totalmente senescentes e com atividade respiratória bastante baixa após quatro dias (Figura 2).

A germinação dos pinhões foi fortemente inibida na temperatura de $2^{\circ} \mathrm{C}$, atingindo cerca de $1 \%$ de germinação após 96 dias de armazenamento (Figura 3A). Na temperatura de $10^{\circ} \mathrm{C}$, houve um significativo incremento na percentagem de germinação, atingindo cerca de 21\% de germinação após 96 dias de armazenamento (Figura 3A). O maior incremento na taxa de germinação dos pinhões foi verificado na temperatura de $20^{\circ} \mathrm{C}$, com cerca de $55 \%$ dos pinhões germinados a partir de 26 dias de armazenamento (Figura 3A). Na temperatura de $30^{\circ} \mathrm{C}$, a percentagem de germinação foi de, no máximo, $9 \%$ aos 26 dias de armazenamento, enquanto pinhões deixados nas temperaturas de 40 e $50^{\circ} \mathrm{C}$ apresentaram taxas de germinação inferior a 1\% (Figura 3A).

A reduzida germinação de pinhões mantidos em temperaturas inferiores a $20^{\circ} \mathrm{C}$ (Figura 3A) reflete a menor atividade metabólica dos tecidos sob refrigeração, como é o caso da respiração (Figuras $1 \mathrm{e}$ 2). A redução na germinação com o aumento da 


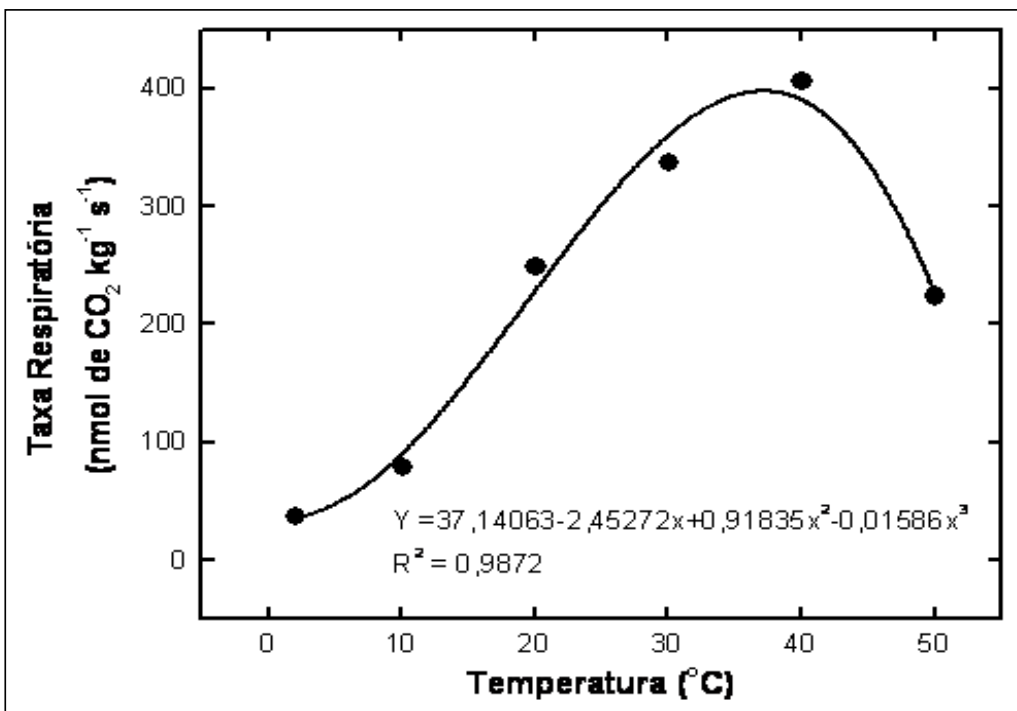

Figura 1 - Relação entre temperatura e taxa respiratória em pinhões, 24h após o armazenamento. viabilidade fisiológica com a desidratação (AQUILA \& FERREIRA, 1984; FARRANT et al., 1989; FOWLER et al., 1998). Portanto, a redução na percentagem de germinação em temperaturas superiores a $20^{\circ} \mathrm{C}$ pode ter sido o resultado da maior desidratação das sementes (Figura 3B), mesmo com a utilização de elevada umidade

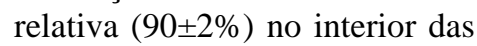
câmaras BOD. Com a elevação da temperatura, mantendo a mesma umidade relativa do ar, ocorre um aumento no déficit de pressão de vapor de água (DPV) entre o pinhão e o ar da câmara. Portanto, o DPV entre o pinhão (com temperatura em equilíbrio com a temperatura de $20^{\circ} \mathrm{C}$ para $30-40^{\circ} \mathrm{C}$ parece refletir o acelerado processo de consumo de reservas, levando à rápida senescência dos tecidos. Na temperatura de $50^{\circ} \mathrm{C}$, inicia-se o processo de desnaturação de enzimas, comprometendo assim o metabolismo dos tecidos (TAIZ \& ZEIGER, 1998), evidenciado pela menor taxa respiratória em relação à temperatura de $40^{\circ} \mathrm{C}$ (Figura 2). Todavia, há de se destacar que os pinhões, em virtude de sua natureza recalcitrante, perdem a temperatura do ar da câmara e cerca de 99,5\% de umidade relativa entre os espaços inter-celulares) e o ar aumenta de 221,5 Pa, na câmara com $20^{\circ} \mathrm{C} / 90 \%$ UR, para $1.168,7$ Pa na câmara com $50^{\circ} \mathrm{C} / 90 \%$ UR. Portanto, o aumento na perda de massa fresca dos pinhões com o aumento na temperatura de armazenamento (Figura 3B) resulta principalmente do aumento na perda de água, como também do aumento no consumo de substratos respiratórios. O aumento na desidratação dos pinhões armazenados em temperaturas superiores

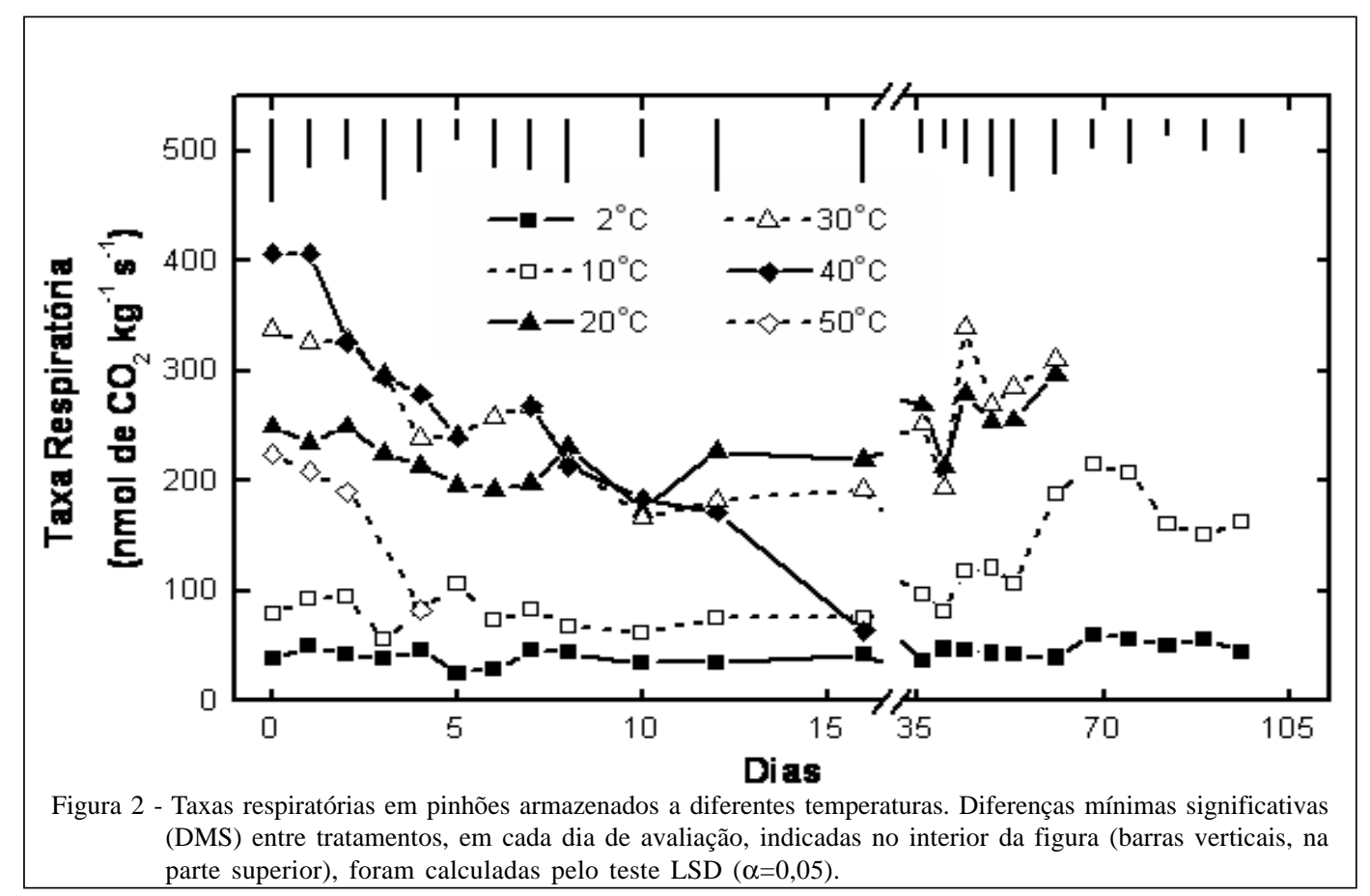

Ciência Rural, v.37, n.2, mar-abr, 2007. 


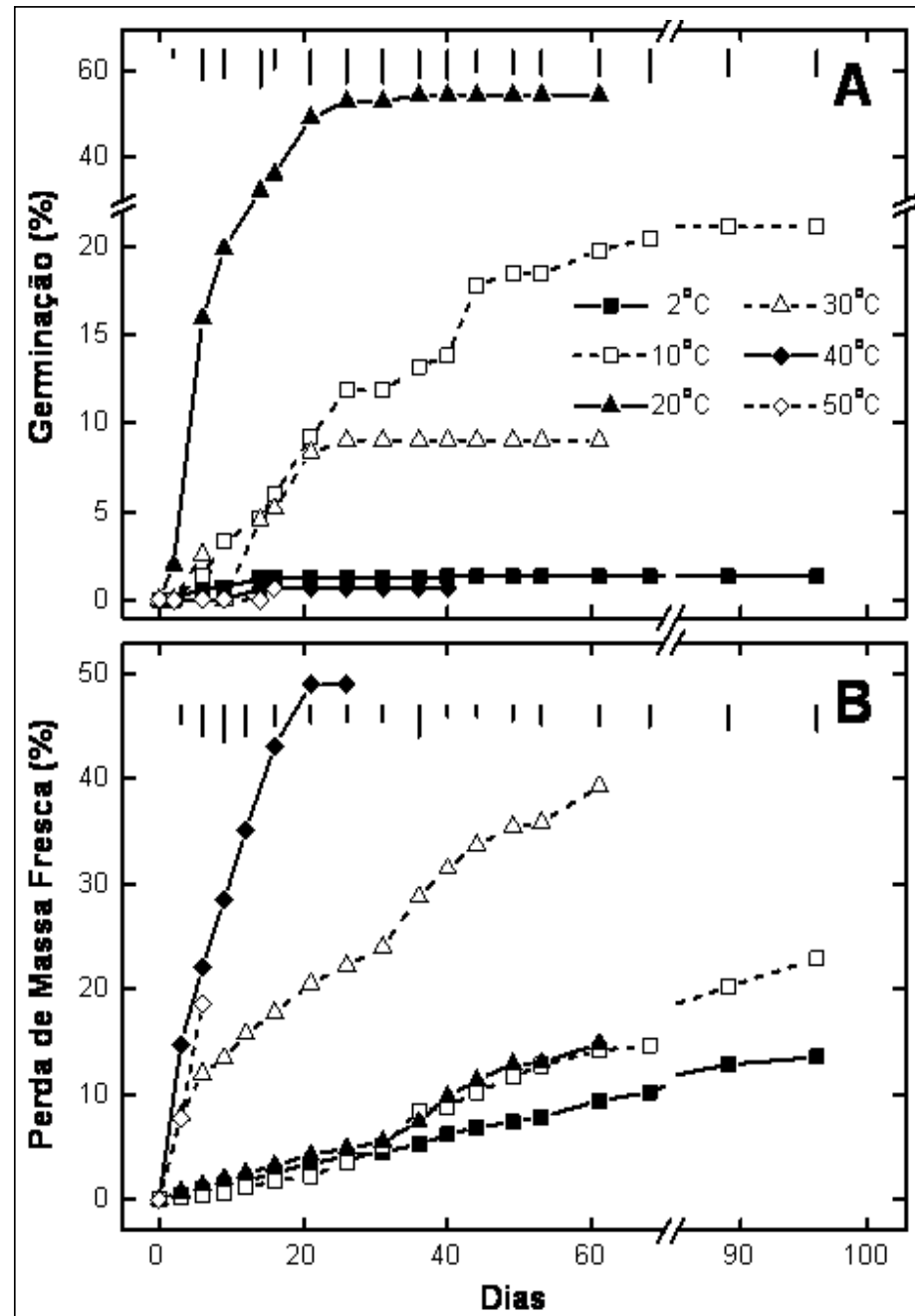

Figura 3 - Percentagens de germinação (A) e de perda de massa fresca (B) em pinhões armazenados a diferentes temperaturas. Diferenças mínimas significativas (DMS) entre tratamentos, em cada dia de avaliação, indicadas no interior da figura (barras verticais na parte superior), foram calculadas pelo teste $\operatorname{LSD}(\alpha=0,05)$. a desidratação e a germinação. O armazenamento em temperaturas elevadas, iguais ou superiores a $20^{\circ} \mathrm{C}$, levou à rápida perda de viabilidade fisiológica, em função do gasto energético com a respiração e da desorganização celular relacionada à desidratação e à senescência dos tecidos. Em temperaturas de $40-50^{\circ} \mathrm{C}$, esses processos foram mais evidentes.

\section{AGRADECIMENTOS}

Ao Conselho Nacional de Desenvolvimento Científico e Tecnológico (CNPq) e à Coordenação de Aperfeiçoamento de Pessoal de Nível Superior (CAPES), pelo apoio financeiro a este projeto.

\section{REFERÊNCIAS}

AQUILA, M.E.A.; FERREIRA. A.G. Germinação de sementes escarificadas de Araucaria angustifolia em solo. Ciência e Cultura, Campinas, v.36, n.9, p.1583-1589, 1984.

BANKS, N.H. et al. Proposal for a rationalized system of units for postharvest research in gas exchange. HortScience, Alexandria, v.30, n.6, p.1129-1131, 1995.

CARVALHO, P.E.R. Araucaria angustifolia (Bertoloni) Otto Kuntze: Pinheiro-do-Paraná. In: CARVALHO, P.E.R. (Ed). Espécies florestais brasileiras: recomendações silviculturais, potencialidades e uso da madeira. Colombo: EMBRAPA-CNPF/ Brasília: EMBRAPA-SPI, 1994. p.70-78.

CORBINEAU, F. et al. Cellular and metabolic events associated with dehydration of recalcitrant Araucaria angustifolia embryos. In: ELLIS, R.H. et al. (Eds). Basic and applied aspects of seed biology. Reding, United Kingdom, 1995. Dordrecht, Netherlands: Kluwer Academic Publishers, 1997. p.715-721.

FARRANT, J.M. et al. Germination-associated events and the desiccation sensitivity of recalcitrant seeds - a study on three unrelated species. Planta, Heidelberg, v.178, p.189-198, 1989.

FOWLER, J.A.P. et al. Conservação de sementes de pinheiro do Paraná sob diferentes condições de ambientes e embalagens. Colombo: EMBRAPA-CNPF, 1998. 4p. (Comunicado Técnico, 34).

Estes resultados demonstram que, ao se armazenar os pinhões visando a sua utilização como alimento, deve-se procurar armazená-los imediatamente após a colheita em temperaturas próximas de $0^{\circ} \mathrm{C}$, em ambiente com elevada umidade relativa, visando a evitar
KLEIN, R.M. Espécies raras ou ameaçadas de extinção do Estado de Santa Catarina. Estudos de Biologia, Curitiba, n.31, p.3-9, 1993. em sementes de Araucaria angustifolia (Bert.) O. Ktze.
RAMOS, A. et al. Alterações bioquímicas e fisiológicas imediatas 
submetidas a secagem em estufa. In: SIMPÓSIO BILATERAL BRASIL-FINLÂNDIA SOBRE ATUALIDADES FLORESTAIS, 1988, Curitiba. Simpósio... Curitiba: Universidade Federal do Paraná, 1988. p.97-110.

SALMEN-ESPINDOLA, L. et al. Cellular and metabolic damage induced by desiccation in recalcitrant Araucaria angustifolia embryos. Seed Science Research, Baton Rouge, v.4, n.2, p.193-201, 1994.

SANTOS, A.J. dos et al. Aspectos produtivos e comerciais do pinhão no Estado do Paraná. Floresta, Curitiba, v.32, n.2, p.163-169, 2002
SAS INSTITUTE (Cary, Estados Unidos). Doing more with SAS/ASSIST software: version 6. Cary, 1990. 789p.

TAIZ, L.; ZEIGER, E. Plant physiology. 2.ed. Sunderland: Sinauer Associates, 1998. 793p.

TOMPSETT, P.B. Manejo e armazenamento de sementes de Agathis spp e Araucaria spp. Silvicultura, São Paulo, v.8, n.30, p.290-293, 1993.

WILLS, R.H. et al. Postharvest, an introduction to the physiology and handling of fruit, vegetables and ornamentals. 4.ed. New York: CAB International, 1998. 262p. 